CARDIOVASCULAR MEDICINE

\title{
Warfarin for non-rheumatic atrial fibrillation: five year experience in a district general hospital
}

\author{
Z R Yousef, S C Tandy, V Tudor, F Jishi, R J Trent, D K Watson, R P W Cowell
}

Heart 2004;90:1259-1262. doi: 10.1136/hrt.2003.023325

See end of article for authors' affiliations

.....................

Correspondence to: Dr Richard Cowell, Department of Cardiology, Wrexham Maelor Hospital, Croesnewydd Road, Wrexham, LL13 7TD, UK; richard.cowell@ new-tr.wales.nhs.uk

Accepted

23 February 2004
Objectives: To assess the long term efficacy of and risks associated with computer aided oral anticoagulation for non-rheumatic atrial fibrillation (NRAF) in a district hospital setting.

Design: Retrospective, age stratified, event driven clinical database analysis.

Setting: District general hospital.

Participants: 739 patients receiving warfarin for NRAF between 1996 and 2001. Patients were selected from an anticoagulation database through appropriate filter settings.

Main outcome measures: Anticoagulation control (international normalised ratio (INR)) and hospitalisations for bleeding complications, thromboembolic events, and stroke.

Results: Over 1484 patient-years, computer assisted anticoagulation was uncontrolled in $38.3 \%$ of patients (INR $<2.0$ or $>3.0$ ). No significant differences in INR control were observed with respect to patient age $(<65,65-75$, and $>75$ years), although to achieve adequate control of anticoagulation, the frequency of testing increased significantly with age. Annual risks of bleeding complications, thromboembolism, and stroke were $0.76 \%, 0.35 \%$, and $0.84 \%$, respectively. No significant differences in these events were observed between the three age groups studied. Patients who had thromboembolic events and haemorrhagic complications were significantly more likely to have been under-anticoagulated $($ INR $<2.0$ ) and over-anticoagulated (INR > 3.0), respectively, at the time of their clinical event.

Conclusions: Computerised long term oral anticoagulation for NRAF in a community setting of elderly and diverse patients is safe and effective. Anticoagulation control, bleeding events, thromboembolic episodes, and stroke rates are directly comparable with those reported in major clinical trials. The authors therefore support the strategy of rate control with long term oral anticoagulation for NRAF in general clinical practice.
This study was conducted at a district general hospital serving a population of 250 000. Geographic factors and the semirural locality preclude migration to nearby hospitals, thus ensuring a stable patient base. The investigation was an event driven retrospective clinical database analysis between September 1996 and September 2001.
The management of NRAF within our unit is based on accepted guidelines. ${ }^{13}$ In particular, our target international normalised ratio (INR) is 2.5 (range 2.0-3.0). INR values $<2.0$ and $>3.0$ indicate suboptimal anticoagulant control, and values $>8.0$ indicate potentially serious breaches requiring urgent treatment. All patients presenting to our unit with stroke or transient neurological deficit while taking warfarin undergo computer tomographic imaging for further assessment.

\section{Anticoagulation database}

Since 1995, all outpatient oral anticoagulation with warfarin at our institution has been centralised and computer assisted. The software (TelePath anticoagulation module vl.3; iSOFT Systems plc, Manchester, UK) uses an accepted protocol (Charles' algorithm). ${ }^{14}$ Frequent internal validations ensure compliance with operational standards.

For each patient, the indication for long term oral anticoagulation, target INR, intended duration of treatment, co-morbidities, and concurrent medication are mandatory input fields. Appropriate database filtering therefore enables precise identification of patient groups.

At each visit, the current INR is compared with the patient's characteristics, previous INR values, and dosing history. These variables are used to generate a schedule

Abbreviations: AFASAK, atrial fibrillation, aspirin, and anticoagulation; BAATAF, Boston area anticoagulation trial for atrial fibrillation; INR, international normalised ratio; NRAF, non-rheumatic atrial fibrillation; PAS, patient administration system; SPINAF, stroke prevention in non-rheumatic atrial fibrillation 
recommending any changes in subsequent warfarin dosage and the next test date.

The database was accessed to identify all patients receiving warfarin for NRAF. Patients receiving treatment for $<12$ months were excluded. For each patient, profiles comprising age at inclusion, sex, number of INR tests, INR values, and dosing intervals were constructed. In addition, episodes where INR values measured $<2.0,>3.0$, and $>8.0$ were identified.

\section{Patient administration system database}

Our hospital subscribes to the national patient administration system (PAS) (iSOFT plc, Manchester, UK), which records all inpatient episodes against a discharge diagnosis (International classification and coding of diseases, 10th revision). Details of patients with NRAF receiving long term oral anticoagulation (obtained from the anticoagulation database) were cross referenced with the PAS database to identify inpatient episodes over the five year study period. For each matching record, hospitalisation dates and discharge diagnoses were recorded. Discharge diagnoses were subsequently categorised with respect to (a) haemorrhagic risks of long term oral anticoagulation (any bleeding event leading to hospitalisation); (b) thromboembolic episodes (transient ischaemic cerebrovascular event, systemic arterial embolism, or pulmonary embolism); and (c) stroke (with radiological confirmation).

\section{Radiology database}

Details of patients presenting with stroke (from the PAS database) were cross referenced with a proprietary radiological database (RadIS; NHS Wales) to determine the aetiology of the cerebral event (ischaemia or haemorrhage).

\section{Statistical analysis}

Age stratified data (patients aged $<65,65-75$, and $>75$ years) are presented as mean (SD). Events are expressed as prevalence within each age group or as an annualised prevalence. Two way analysis of variance with Bonferroni post hoc testing was used for parametric between group comparisons. Fisher's exact test was used to compare nonparametric data (InStat v3.01; GraphPad Software Inc, San Diego, California, USA). Significance was considered to be $\mathrm{p}<0.05$.

\section{RESULTS}

There were 739 patients with NRAF receiving long term oral anticoagulation for $\geqslant 12$ months during the five year study period, for a total of 1484 patient-years of treatment. Their mean (SD) age was 73.1 (4.0) (range 21-97 years) and 52.1\% were men. One hundred and forty nine patients $(20.2 \%)$ were aged $<65$ years, 284 (38.4\%) were aged 65-75 years, and 306 $(41.4 \%)$ were aged $>75$ years. The prevalence of female sex increased from $33 \%$ of patients aged $<65$ years to $55 \%$ of patients $>75$ years $(\mathrm{p}<0.01)$.

\section{Anticoagulation control}

During the five year study period, 27026 INR tests to monitor anticoagulant control were performed. The frequency of testing increased significantly and the interval between tests shortened significantly with advancing age (table 1$)$. Table 1 shows the mean (SD) INR for all patients $(2.43(0.23))$ and age related mean INR values. Overall, $22.9 \%$ of INRs measured $<2.0,14.3 \%$ measured $>3.0$, and in $37.2 \%$ of cases, anticoagulation was uncontrolled (INR $<2.0$ or $>3.0$ ). No significant differences in anticoagulation control were observed with respect to a patient's age (table 1).

\section{Clinical events}

Figure 1 shows haemorrhagic complications of long term oral anticoagulation requiring hospitalisation $(\mathrm{n}=28)$, thromboembolic events $(\mathrm{n}=13)$, and stroke $(\mathrm{n}=31)$. No significant differences in bleeding complications and embolic events were demonstrable between the three age groups. Similarly, the annualised combined event rate of bleeding complications, thromboembolic events, and stroke was similar in patients aged $<65(1.48 \%), 65-75$ (1.97\%), and $>75$ years $(2.16 \%$ ) (fig 1$)$.

\section{Clinical events versus anticoagulation control}

Figure 2 shows INR control at the time of clinical events. Compared with the study group as a whole, patients who had thromboembolic and cerebrovascular events were significantly more likely to have been under anticoagulated (INR $<2.0)$ at the time of the event $(69.2 \% \vee 22.9 \%$, p $<0.01$; $58.1 \% \vee 22.9 \%, \mathrm{p}<0.05$, respectively). Similarly, the prevalence of overanticoagulation (INR > 3.0) was greater in patients experiencing a haemorrhagic complication than in the rest of the study group $(42.9 \% v 14.3 \%$, p $<0.05)$. During the five year study, 24 episodes where the INR measured $>8.0$ were recorded. The prevalence of INR readings $>8.0$ was similar across the three patient groups $(<65$ years, $\mathrm{n}=7(4.7 \%) ; 65-75$ years, $\mathrm{n}=9(3.2 \%)$; and $>75$ years, $\mathrm{n}=8(2.6 \%))$.

\section{DISCUSSION}

Over nearly 1500 patient-years of treatment in a community setting, we have shown that computer assisted dosing of warfarin can be safe and effective. In addition, we have observed that outcomes reported in clinical trials examining the use of long term oral anticoagulation in NRAF are reproducible in general clinical practice.

To date, five major randomised clinical studies of long term oral anticoagulation in NRAF have been conducted. ${ }^{5-9}$ Although the studies differed with respect to patient numbers, entry criteria, levels of anticoagulation, control group treatment (aspirin or no antiplatelet treatment), and

Table 1 Patient demographics and international normalised ratio (INR) tests

\begin{tabular}{|c|c|c|c|c|c|c|c|c|c|}
\hline \multirow[b]{2}{*}{$\begin{array}{l}\text { Age group } \\
\text { (years) }\end{array}$} & \multirow[b]{2}{*}{$\mathbf{n}$} & \multirow[b]{2}{*}{$\begin{array}{l}\text { Mean age } \\
\text { (years) }\end{array}$} & \multirow[b]{2}{*}{ Men } & \multicolumn{3}{|l|}{ INR tests } & \multicolumn{3}{|c|}{ INR control } \\
\hline & & & & Mean INR & $\begin{array}{l}\text { Number/ } \\
\text { patient/year }\end{array}$ & $\begin{array}{l}\text { Dosing interval } \\
\text { (days) }\end{array}$ & $<\mathbf{2 . 0}$ & $>3.0$ & $<2.0$ or $>3.0$ \\
\hline$<65$ & 149 & $56.4(7.8)$ & $67.1 \%$ & $2.4(0.3)$ & $17.4(5.1)$ & $21.0(2.6)$ & $25.3 \%$ & $12.7 \%$ & $37.9 \%$ \\
\hline $65-75$ & 284 & $69.8(2.7)$ & $63.0 \%$ & $2.4(0.2)$ & $36.8(6.7)$ & $9.9(2.2)$ & $22.2 \%$ & $14.2 \%$ & $36.4 \%$ \\
\hline$>75$ & 306 & $80.3(2.8)$ & $45.4 \%$ & $2.5(0.3)$ & $45.7(6.0)$ & $8.1(6.9)$ & $22.3 \%$ & $15.4 \%$ & $37.7 \%$ \\
\hline
\end{tabular}

Data are mean (SD) or percentage.

Number of INR tests increased significantly with age: $<65 v 65-75$ years $(p<0.01)$ and $65-75 v>75$ years $(p<0.01)$. Correspondingly, the interval between tests shortened significantly with increasing age: $<65 v 65-75$ years $(p<0.01)$ and $65-75 v>75$ years $(p<0.01)$. No significant between group differences were observed with respect to INR control. 


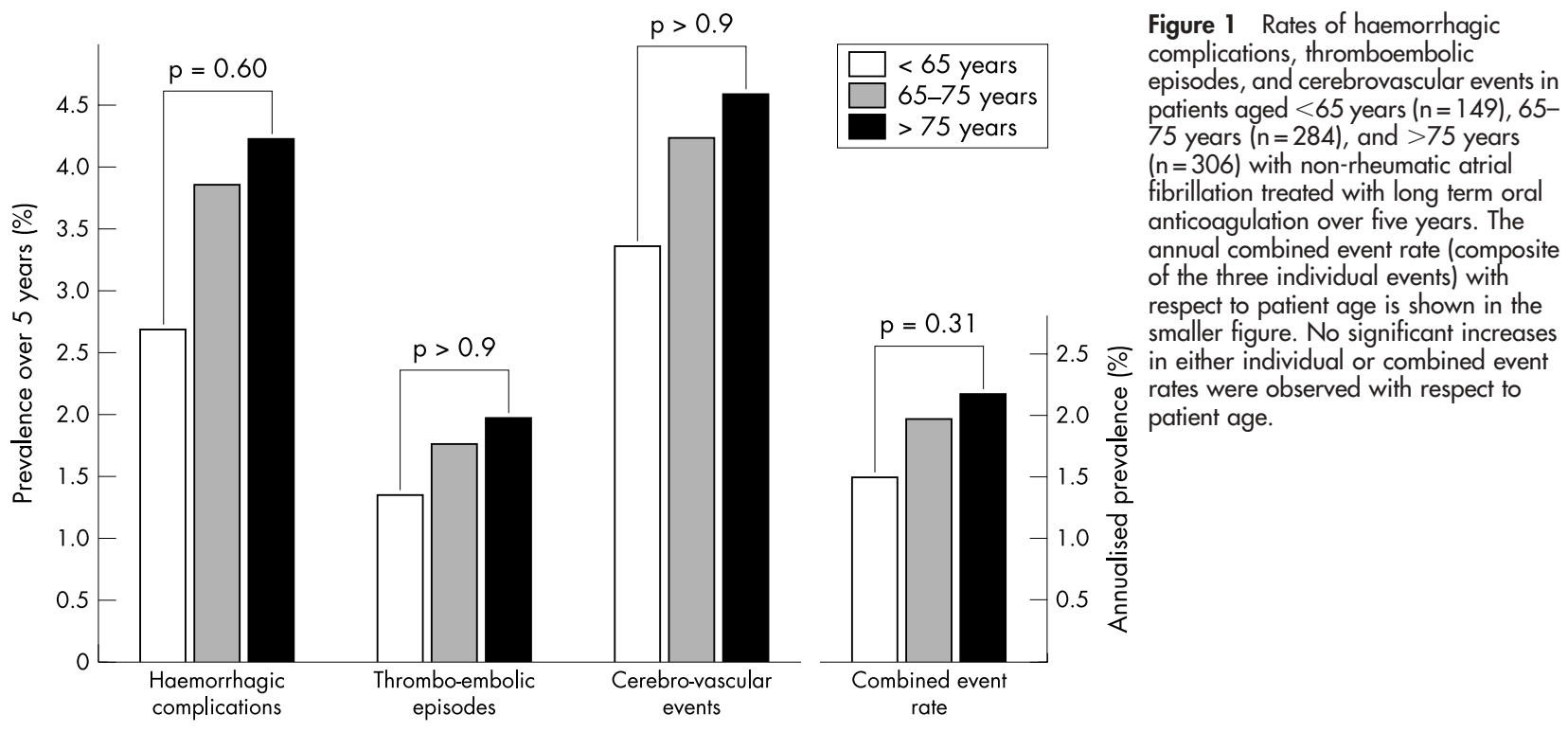

end points (combined and individual), all studies were terminated prematurely after significant benefits were shown for patients receiving long term oral anticoagulation. Table 2 compares isolated end points-anticoagulation control, bleeding complications, thromboembolic events, and ischaemic strokes-from these trials with our experience (table 2).

Patients in the present study were more representative of the general population compared with those recruited to clinical trials. For example, patients in the current investigation were on average 4-8 years older (mean age 73 years $v 65-$ 69 years $^{5-8}$; only median age was reported in the AFASAK (atrial fibrillation, aspirin, and anticoagulation) study ${ }^{9}$ ). More of our patient population were women (48\% v 24$47 \%)^{5-7}$; SPINAF (stroke prevention in non-rheumatic atrial fibrillation $)^{8}$ was an all male study.

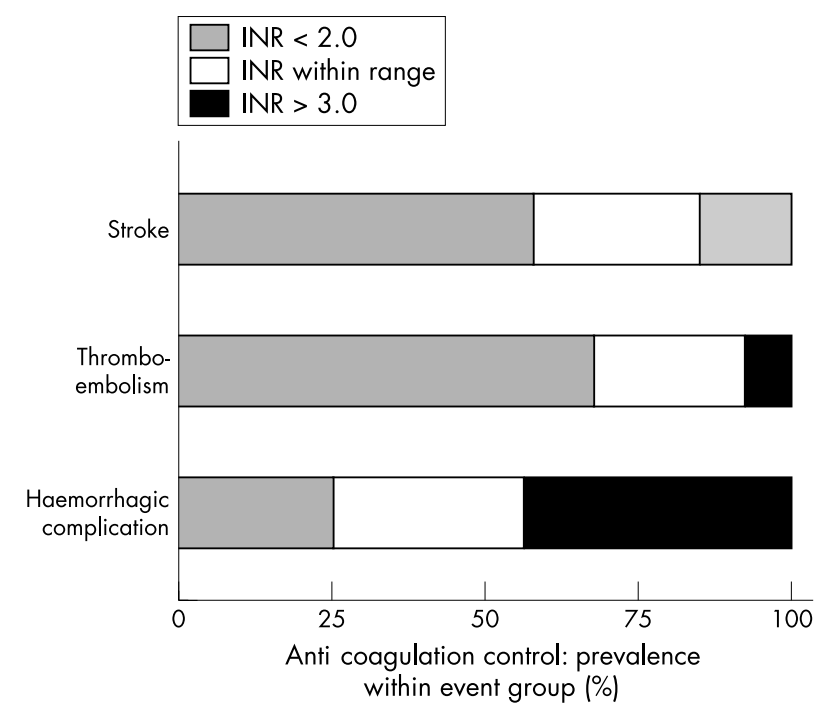

Figure 2 Prevalence of under-anticoagulation (INR $<2.0$ ), overanticoagulation (INR > 3.0), and correct anticoagulation (INR within range 2.0-3.0) at the time of each clinical event. Patients who had a stroke or thromboembolic event were more likely to have an INR $<2.0$ (58.1\% and $69.2 \%$, respectively). Patients experiencing a haemorrhagic complication were more likely to have been relatively overanticoagulated (INR > 3.0; 42.9\%).
Annual ischaemic stroke rates observed in the present study are comparable with the rates reported from clinical trials $(0.84 \% v 0.4-2.5 \%) .^{5-9}$ This is an important observation suggesting that in this setting, clinical trial data (involving selected and relatively young patients) may be extrapolated to the general population. At the same time, haemorrhagic complications observed in the current investigation $(0.76 \%$ a year) were less frequent than expected $(7-18.5 \%$ a year $) .^{5-9}$ This difference may relate to variable trial definitions of major and minor bleeding and the methods we used, which recognised only hospitalised events. Our observed prevalence of thromboembolic events (including transient ischaemic cerebral events) is comparable with the rates reported in clinical trials $\left(0.35 \vee 0-1.6 \% /\right.$ year $\left.^{5-9}\right)$ and probably reflects the fact that most of these events result in hospitalisation. Trial data regarding thromboembolic events, however, are deficient, since embolic episodes were not reported in BAATAF (Boston area anticoagulation trial for atrial fibrillation), ${ }^{5}$ no events were observed in AFASAK, ${ }^{9}$ and embolic rates for the remaining studies $^{6-8}$ have been derived from composite outcomes.

Our anticoagulation control (38\% uncontrolled) is directly comparable with the levels experienced in trials (uncontrolled range $17-56 \%) .^{5-9}$ To achieve these levels, we found that the frequency of testing and intervals between tests had to be more rigorous for the elderly. This probably relates to compliance issues ${ }^{15}$ and increasing polypharmacy ${ }^{16}$ among patients of advancing age.

Where INR values were beyond the target range, they tended to be $<2.0$, rather than $>3.0$. This is a feature of our software with in-built protocols that tend to underdose rather than overdose warfarin. ${ }^{14}$ Although this policy safeguarded against the development of haemorrhagic complications, underanticoagulated patients were predisposed to a threefold increased risk of a thromboembolic event (fig 2). Although initially alarming, these data are reassuring, since they endorse the value of long term oral anticoagulation (target INR of 2.5) in this group of patients.

\section{Study limitations}

This study has several limitations. Firstly, our methods allowed only for the identification of hospitalised episodes to our institution. As a result, we were apt to miss events managed at home or at another hospital and out of hospital 
Table 2 Results of landmark randomised clinical trials of long term oral anticoagulation for non-rheumatic atrial fibrillation compared with a five year experience in a district general hospital (DGH)

\begin{tabular}{|c|c|c|c|c|c|c|c|c|c|c|c|c|}
\hline & \multicolumn{2}{|c|}{ BAATAF $_{5}$} & \multicolumn{3}{|c|}{ AFASAK $^{9}$} & \multicolumn{2}{|l|}{ SPINAF $^{8}$} & \multicolumn{2}{|l|}{ SPAF- $1^{6}$} & \multicolumn{2}{|l|}{ CAFA $^{7}$} & \multirow{2}{*}{$\frac{\mathrm{DGH}}{\mathrm{W}}$} \\
\hline & w & C & W & C & C & W & C & w & C & w & C & \\
\hline Number & 212 & 208 & 335 & 336 & 337 & 260 & 265 & 210 & 211 & 187 & 191 & 739 \\
\hline Age (years) & $69(9)$ & $68(9)$ & - & - & - & $67(7)$ & $67(7)$ & 65 & 66 & $68(9)$ & $67(10)$ & $73(4)$ \\
\hline Men & $75 \%$ & $70 \%$ & $53 \%$ & $55 \%$ & $54 \%$ & $100 \%$ & $100 \%$ & $74 \%$ & $70 \%$ & $76 \%$ & $73 \%$ & $52 \%$ \\
\hline \multicolumn{13}{|l|}{ INR control } \\
\hline Range & $1.5-2.7$ & \pm Aspirin & $2.8-4.2$ & Aspirin & Placebo & $1.4-2.8$ & Placebo & $2.0-3.5$ & Placebo & $2.0-3.0$ & Placebo & $2.0-3.0$ \\
\hline Under target & $8 \%$ & & $26 \%$ & & & & & & & $40 \%$ & & \\
\hline Over target & $9 \%$ & & $1 \%$ & & & $15 \%$ & & $5 \%$ & & $17 \%$ & & $15 \%$ \\
\hline Uncontrolled & $17 \%$ & & $27 \%$ & & & $44 \%$ & & $28 \%$ & & $57 \%$ & & $39 \%$ \\
\hline \multicolumn{13}{|c|}{ Outcomes (\%/year) } \\
\hline Bleeding episodes & 8.58 & 6.97 & 7.00 & 2.00 & 2.00 & 11.40 & 15.30 & 1.50 & 1.60 & 18.50 & 9.50 & 0.76 \\
\hline Embolic events & - & - & 0.00 & 1.34 & 1.31 & 1.56 & 2.25 & 1.10 & 2.06 & 1.00 & 1.90 & 0.35 \\
\hline Stroke & 0.41 & 2.98 & 2.00 & 4.13 & 4.19 & 0.90 & 4.30 & 2.30 & 6.99 & 2.50 & 4.26 & 0.84 \\
\hline
\end{tabular}

AFASAK, atrial fibrillation, aspirin, and anticoagulation; BAATAF, Boston area anticoagulation trial for atrial fibrillation; C Control; CAFA, Canadian atrial fibrillation anticoagulation; SPAF, stroke prevention in atrial fibrillation; SPINAF, stroke prevention in non-rheumatic atrial fibrillation; W, warfarin.

deaths. Since the outcomes under investigation were relatively infrequent (six bleeding events, three embolic episodes, and six strokes each year) even minor discrepancies in true versus observed events may appreciably affect our results. Secondly, we have not compared survival between patients, since we cannot be confident that our data acquisition as described would have included all deaths. Drop out rates, however, remained constant throughout the five year study period: about $10 \%$ attrition of patients from the anticoagulation database each year. Thirdly, it is accepted that PAS entries for discharge diagnoses can be inaccurate. ${ }^{17}$ The events examined in the current study, however, are unambiguous; thus, we believe that false coding errors (if any) would not significantly alter our findings. Lastly, only $7 \%$ of patients in the present study were aged $>85$ years. Patients must therefore have been intuitively screened clinically before long term oral anticoagulation was initiated, restricting the use of warfarin to the fittest elderly patients. Our data (especially for the very elderly) must therefore be viewed in the context of standard clinical practice.

\section{Conclusions}

Long term administration of warfarin for NRAF based on computer assisted protocols in the community is safe and effective. Our experience approaching 1500 patient-years suggests that satisfactory anticoagulant control is possible ( $60 \%$ of measures within target range) and comparable with levels reported from landmark clinical trials.

Assuming control group event rates in clinical trials are also applicable, long term oral anticoagulation for NRAF in general clinical practice remains a highly beneficial treatment, with annual bleeding, thromboembolic event, and stroke rates of $0.76 \%, 0.35 \%$, and $0.84 \%$, respectively.

We therefore endorse the practice of computer aided dosing of warfarin and recommend its wider use for NRAF in the community, which includes elderly and heterogeneous patients after appropriate clinical assessment.

\section{Authors' affiliations}

Z R Yousef, F Jishi, R J Trent, R P W Cowell, Department of Cardiology, Wrexham Maelor Hospital, Croesnewydd Road, Wrexham, UK S C Tandy, V Tudor, D K Watson, Department of Haematology, Wrexham Maelor Hospital

\section{REFERENCES}

National Heart, Lung, and Blood Institute Working Group on Atria Fibrillation. Atrial fibrillation: current understandings and research imperatives. J Am Coll Cardiol 1993;22:1830-4.

2 Go AS, Hylek EM, Phillips KA, et al. Prevalence of diagnosed atrial fibrillation in adults: national implications for rhythm management and stroke prevention: the anticoagulation and risk factors in atrial fibrillation (ATRIA) study. JAMA $2001 ; 285: 2370-5$.

3 Feinberg WM, Blackshear JL, Laupacis A, et al. Prevalence, age distribution, and gender of patients with atrial fibrillation.: analysis and implications. Arch Intern Med 1995; 155:469-73.

4 Orsinelli DA. Current recommendations for the anticoagulation of patients with atrial fibrillation. Prog Cardiovasc Dis 1996;39:1-20.

5 Boston Area Anticoagulation Trial for Atrial Fibrillation Investigators. The effect of low-dose warfarin on the risk of stroke in patients with nonrheumatic atrial fibrillation. The Boston area anticoagulation trial for atrial fibrillation investigators. N Engl J Med 1990;323:1505-11.

6 Anon. Stroke prevention in atrial fibrillation study: final results. Circulation 1991;84:527-39.

7 Connolly SJ, Laupacis A, Gent M, et al. Canadian atrial fibrillation anticoagulation (CAFA) study. J Am Coll Cardiol 1991; 18:349-55.

8 Ezekowitz MD, Bridgers SL, James KE, et al. Warfarin in the prevention of stroke associated with nonrheumatic atrial fibrillation. Veterans Affairs Stroke Prevention in Nonrheumatic Atrial Fibrillation Investigators. N Engl J Med 1992;327:1406-12.

9 Petersen P, Boysen G, Godtfredsen J, et al. Placebo-controlled, randomised trial of warfarin and aspirin for prevention of thromboembolic complications in chronic atrial fibrillation. The Copenhagen AFASAK study. Lancet 1989;i:175-9.

10 Van Gelder IC, Hagens VE, Bosker HA, et al. A comparison of rate control and rhythm control in patients with recurrent persistent atrial fibrillation. N Engl J Med 2002;347:1834-40.

11 Wyse DG, Waldo AL, DiMarco JP, et al. A comparison of rate control and rhythm control in patients with atrial fibrillation. N Engl J Med 2002;347: 1825-33.

12 Boos CJ, More RS, Carlsson J. Persistent atrial fibrillation: rate control or rhythm control: rate control is not inferior to rhythm control. BMJ 2003;326:1411-2.

13 Fuster V, Ryden LE, Asinger RW, et al. ACC/AHA/ESC guidelines for the management of patients with atrial fibrillation: executive summary. A report of the American College of Cardiology/American Heart Association task force on practice guidelines and the European Society of Cardiology committee for practice guidelines and policy conferences (committee to develop guidelines for the management of patients with atrial fibrillation) developed in collaboration with the North American Society of Pacing and Electrophysiology. Circulation 2001;104:2118-50.

14 Wilson $\mathbf{R}$, James $\mathrm{AH}$. Computer assisted management of warfarin treatment. BMJ 1984;289:422-4.

15 Wandless I, Davie JW. Can drug compliance in the elderly be improved? BMJ 1977;i:359-61.

16 Veehof LJ, Stewart RE, Meyboom-de Jong B, et al. Adverse drug reactions and polypharmacy in the elderly in general practice. Eur J Clin Pharmacol 1999;55:533-6.

17 Williams JG, Mann RY. Hospital episode statistics: time for clinicians to get involved? Clin Med 2002;2:34-7. 